\title{
BOOTHMATES FOREVER? - ON TEAMWORK IN A SIMULTANEOUS INTERPRETING BOOTH
}

\author{
AGNIESZKA CHMIEL
}

Abstract: Simultaneous interpreting (SI) is a cognitively demanding task. This is why there are typically two interpreters working in a booth and taking turns every 30 minutes or so. Interpreters work in pairs not only to be able to overcome fatigue, but also to cooperate and help each other. This article is an attempt to shed some light on the process of booth teamwork. Cooperation in the booth is examined in the professional context, which leads to conclusions regarding the incorporation of this skill in conference interpreter training.

A survey was conducted among 200 free-lance interpreters associated in AIIC and working on various markets to find out more about their expectations and needs as regards assistance from their booth partners. The respondents were asked about their mode of operation, activities in the booth when off-mike and their perception of the need to teach cooperation to interpretation trainees. It turns out that there are some factors that may impede teamwork in the simultaneous interpreting booth. Interpreters who are off-mike can engage in last-minute preparation using materials supplied by the organizers shortly before the commencement of a conference. Additionally, fatigue may prevent them from actively listening to the input and assisting their boothmate. The results of the survey may help answer the question if teamwork and turn-taking should be part of simultaneous interpreting courses.

Keywords: simultaneous interpreting, cooperation, interpreter training, booth manners

\section{INTRODUCTION}

Conference interpreting is a challenging task in terms of cognitive processing. It poses strong demands on the interpreter's memory and attention. Interpreters usually work near saturation level (Gile 1999), which means that their available cognitive resources are sometimes insufficient for appropriate processing and interpretation of the incoming speech. Additionally, this mentally challenging work is performed under stress and time constraints, which leads to fatigue. These extraordinary demands of the interpreting task have made it necessary to ensure special working conditions for SI, with at least two interpreters present in the booth. According to the professional standards devised by AIIC (the International Association of Conference Interpreters): "An interpreter shall not, as a general rule, work alone in a simultaneous interpretation booth, without the availability of a colleague to relieve her or him should the need arise" (AIIC: 2008). In the simplest conference setting involving two languages, two bidirectional interpreters 
should be employed to work as a team in one booth. Moreover, "given the constraints related to quality and health, the normal duration of an interpreter's working day shall not exceed two sessions of between two-anda-half and three hours each" (AIIC: 2008). Thus, a typical setup for simultaneous interpreting involves two interpreters in a booth, taking turns every 30 minutes or so for a maximum of about three hours at a time, which is considered the maximum period for sustaining the necessary concentration and accuracy (Nolan 2005: 4).

Interpreters work in pairs not only to take turns and relax, but also to cooperate and help each other. The relations between interpreters working in the same booth are jocularly referred to as konkabinat in Polish. The word is a pun on two words: konkubinat (common-law marriage, cohabitation) and kabina (booth). To what extent do boothmates cooperate and rely on each other? The study described below has been devised to shed more light on cooperation in the simultaneous interpreting booth. The author's experience as a conference interpreter and interpretation trainer has led to the following question: should we teach cooperation and all the elements thereof to interpreting trainees? Examination of current practices applied by professional interpreters on various markets seems necessary to provide the answer. This may further entail changes in training to adjust it to market expectations so that graduates of conference interpretation training programmes are ready to embark on their professional careers and work with more experienced colleagues. Courses or modules devoted especially to professional practice have already been incorporated in some current M.A. programs in interpreting (for instance: "Conference interpreting as a profession" - a 30-hour course offered as part of interpreter training at AMU, Poznan', Poland; professional ethics and standards taught as part of the Simultaneous Interpreting course at Middlesex University or booth etiquette as listed among course contents of the M.A. Conference Interpreting Techniques program at the University of Westminster, London, England).

\section{REFERENCES TO TEAMWORK IN SI LITERATURE}

Many conference interpreting trainers focus in their courses mainly on individual skills (such as anticipation, reformulation, self-monitoring, terminology development, information processing subtasks). Having analysed 
This is an Author's Accepted Manuscript of an article in Across Languages and Cultures 9 (2), pp. 261-276 (2008), DOI: 10.1556/Acr.9.2008.2.6, available here:

http://www.akademiai.com/content/b512262w457x1210/?p=2ef008acd8774ea1b0a44cf0df68f970\&pi=5

linguistic issues in the trainee's output, they may also comment on less important extra-linguistic features such as delivery and voice quality. Cooperation in the booth seems much less important and this is reflected in the literature on simultaneous interpretation training. Many researchers rightly focus on the interpreter's individual skills and there are few references to teamwork in the literature on simultaneous interpreting. For instance, Jones mentions writing down numbers as the area where there should be real teamwork in the booth (2002: 119). Kalina considers "team strength and composition" to be a peri-process factor affecting the quality of conference interpreting (2001: 27). Gile lists "using the boothmate's help" as one of the reformulation tactics and claims that "the presence of a passive interpreter in the booth is (...) a major asset to the active interpreter" (1995: 196). TaylorBouladon points to the importance of personal interactions between work colleagues by claiming that the interpreter's team becomes his/her family for the duration of the conference. She focuses on team spirit and solidarity because interpreters are judged as a team. Assistance is a sensitive issue and rules should be stated clearly before the interpreting begins. Unsolicited assistance may do more harm than indifference (Taylor-Bouladon 2007).

Jensen, who is a typical practisearcher, discusses aspects of a strategic partnership in a booth (2008). Good cooperation between interpreters can increase quality of the output and client satisfaction. Jensen stresses consistency of terminology and avoidance of behaviours that annoy the partner (2008: 1). A problematic relationship arises when interpreters differ in their abilities. In such a case, the less-qualified partner should contain anxiety and be open to professional advice from the more experienced colleague. The latter, on the other hand, should avoid resentment and show tolerance (Jensen 2008: 2). Psychological differences between partners are also of significance, but these are a crucial factor in any team work in any profession. Like TaylorBouladon, Jensen underlines the importance of team spirit and negotiations. Booth partners should negotiate not only if, how and when assistance should be offered, but also when to take turns and how to time the turns. In his smallscale survey involving only thirteen interpreters, Jensen found that the majority of professionals expected their partners to stay in the booth and help when off-mike. The preferred form of assistance was written notes and the average duration of a turn would be 30 minutes. Switches should be made at natural breaks without a mechanical timer (Jensen 2008: 8). He also lists 
This is an Author's Accepted Manuscript of an article in Across Languages and Cultures 9 (2), pp. 261-276 (2008), DOI: 10.1556/Acr.9.2008.2.6, available here:

http://www.akademiai.com/content/b512262w457x1210/?p=2ef008acd8774ea1b0a44cf0df68f970\&pi=5

annoying behaviours, such as cutting off by taking the mike, making faces, eating and doing personal hygiene (selected answers with the most frequent given first) (Jensen 2008: 8).

It seems that not much has been written on cooperation in the booth by Interpreting Studies scholars. Apart from the sources referred to above, brief comments on the issue are found in Nolan (2005) and Gillies (2004). Most of the authors writing about booth relations are practicing conference interpreters themselves. They provide advice based on their own professional experience and use it in training. Booth teamwork is part of conference interpreter training - students are made aware that an interpreter who is offmike should attend to the speaker's message in order to assist his/her colleague by writing down non- contextual information or by searching for terminology.

Theory sometimes parts company with practice and in the practice of SI there are several factors that may impede teamwork in the booth. Interpreters who are off-mike may engage in last-minute preparation using materials supplied by the organisers shortly before the commencement of a conference. Additionally, fatigue may prevent them from actively listening to the input and assisting their boothmate. Therefore, a study was conducted to check if and to what extent interpreters cooperate with each other in the booth. The details will be discussed in the subsequent sections.

\section{MAPPING THE REALITY - AN ONLINE QUESTIONNAIRE FORM}

The choice of research method for examining cooperation in the booth was rather limited. An observation method (video recording of interpreters working in a booth) was rejected since it would be very hard to obtain sufficient material for analysis. A recording of one working day at one conference would suffice for a case study only. The aim of the project was to examine solutions applied widely by the professional community (a norm in Chesterman's understanding of the concept, Chesterman 1997), so it was necessary to involve a high number of subjects in the study. Thus, introspection seemed the only viable methodology in this case. Hence, a questionnaire was devised and sent to professional interpreters with questions 
about the amount and type of assistance they provide to and expect from their colleagues. Some questions also pertained to turn-taking techniques and activities simultaneous interpreters engage in while their booth partners are on mike. The results should shed more light on the doings of non-active boothmates and help answer the question if teamwork and turn-taking should be part of simultaneous interpreting courses.

Requests to participate in the survey as respondents were sent by e-mail to 1,000 professional interpreters. All potential respondents were AIIC members with English as one of their working languages (not necessarily A). Messages were individual and personal - they were sent to single addressees (to avoid rejection of an e-mail message with multiple addresses as spam). Freelance rather than in-house interpreters were selected. The reason was that the study was aimed at examining solutions used by individual interpreters working for various clients and not enforced by a given employer (such as the UN or the European Commission). The potential respondents came from various countries (Argentina, Australia, Austria, Brazil, Canada, Denmark, Finland, France, Germany, Greece, Hong Kong, Ireland, Italy, Japan, Mexico, Netherlands, Norway, Spain, Sweden, Switzerland, UK, USA, Venezuela). However, the exact geographical distribution of actual respondents was not controlled for technical reasons.

To facilitate data processing, the questionnaire was prepared as an online form with various types of questions. Close-ended questions offered a preexisting set of answers displayed as a pop-down menu or were presented as traditional multiple- choice questions (with radio buttons or checkboxes). Open-ended questions were limited to the minimum so as not to discourage respondents from completing the form. No character limit was set for text fields in open-ended questions to allow ample space for replies from interpreters willing to share more thoughts on the subject.

The e-mail messages sent to potential respondents explained the rationale behind the survey and included a link to the website with the online form. Since some interpreters might have been wary of following the link for fear of computer viruses, the questionnaire was also included in the message body. In fact, some respondents chose this form of reply. Thus, the solution seems appropriate and no replies were lost due to the survey format. 
This is an Author's Accepted Manuscript of an article in Across Languages and Cultures 9 (2), pp. 261-276 (2008), DOI: 10.1556/Acr.9.2008.2.6, available here:

http://www.akademiai.com/content/b512262w457x1210/?p=2ef008acd8774ea1b0a44cf0df68f970\&pi=5

The response rate is always awaited with anxiety by researchers trying to elicit responses in survey. The requests were sent to as many as 1,000 interpreters because a much smaller number of responses were expected. The response rate in the study was approx. 20\%, which means that the results presented below are based on a questionnaire completed by 200 respondents.

\subsection{Respondent Profile}

The first questions of the survey were introductory and aimed at specifying the profile of the respondent. Although all respondents described themselves as free- lancers in the AIIC database of members, some of them identified themselves as in- house interpreters in the survey. Such answers (not more than 5) were rejected in order not to skew the results. Thus, all respondents considered in the study were freelance AIIC interpreters with approximately 25 years of experience (ranging from 6 to 52). This means that the data in the study have high reliability, since they come from people who really know the profession and shape their working environment. $82 \%$ of all respondents received formal training in conference interpreting. The survey showed that interpreters very rarely work with one colleague only. $75 \%$ of them work with various colleagues in the booth and it does not matter to $71 \%$ of them if they have to work with an unknown colleague. Most of the respondents (86\%) were bi-directional interpreters (working into their B language).

\subsection{The Questions}

The survey was divided into clear sections devoted to assistance in the booth, the activities of the passive interpreter, turn-taking, directionality and active/passive control of input when off mike. The section on directionality was included for the purpose of a separate study and will not be analysed in this paper. (The whole questionnaire constitutes Appendix A.)The first part pertained to the type of assistance expected from a booth partner and provided to a booth partner if asked. Since the boothmate's help may be more needed in specific contexts (such as processing of non-contextual information names, statistics), there were detailed questions focused on assistance with numbers, units of measure, names, place names, searching for a document referred to by the speaker, finding terminology, and offering and criticism. The second part, on the activities of the passive interpreter offered some 
This is an Author's Accepted Manuscript of an article in Across Languages and Cultures 9 (2), pp. 261-276 (2008), DOI: 10.1556/Acr.9.2008.2.6, available here:

http://www.akademiai.com/content/b512262w457x1210/?p=2ef008acd8774ea1b0a44cf0df68f970\&pi=5

potential answers and a text field for additional response. The turn-taking questions addressed such issues as how often and in what manner switching is done and whether it should be included in the interpreter training programmes. The last part examined the effect of fatigue on the activities of the passive interpreter. Since it is always difficult to foresee all the potential issues that may arise in relation to the topic under study, a text box was included at the bottom of the survey form to elicit any additional comments.

\section{RESULTS AND DISCUSSION}

\subsection{Assistance and Expectations}

The survey shows that the majority of interpreters expect their booth partners to assist them first of all by searching for a document needed $-88 \%$ of positive answers (15\%-always, $31 \%$-often, $42 \%$-sometimes), by writing down numbers $-81 \%$ of positive responses (11\%-always, $29 \%$-often, $41 \%$ sometimes) and by supplying a term missing in the active interpreter's output - 74\% (8\%-always, 22\%- often, 44\%-sometimes). The least expected categories of assistance are writing down place names $-53 \%$ of negative responses (22\%-rarely, 22\%-very rarely, 9\%- never) and names - $44 \%$ of negative answers (20\%-rarely, 18\%-very rarely, 6\%- never). The question about assistance in the form of pointing out mistakes was quite interesting. It seems that $57 \%$ of interpreters are prepared to listen to critical comments provided by their work colleagues, while $9 \%$ do not expect that type of assistance. Aggregated results are presented in Figure 1 below.

Figure 2 shows total results for questions about type of assistance offered by professional conference interpreters. It happens much more often that interpreters are willing to provide assistance than they actually need and expect it. This might be related to their high expertise, acquired over years of practice. When off mike, most of them would assist the active interpreter by searching for documents (61\% of "always" answers), writing down numbers (60\% of "always" answers), providing units of measure (50\% of "always" answers) and terminology (48\% of "always" answers). However, they are much less willing to point out their colleague's mistakes $-10 \%$ would never do that, $20 \%$ do it very rarely and $17 \%$ do it rarely. The approach to critical remarks is a very personal characteristic and interpreters, unsure of the 
This is an Author's Accepted Manuscript of an article in Across Languages and Cultures 9 (2), pp. 261-276 (2008), DOI: 10.1556/Acr.9.2008.2.6, available here:

http://www.akademiai.com/content/b512262w457x1210/?p=2ef008acd8774ea1b0a44cf0df68f970\&pi=5

partner's reaction, might simply want to avoid critical remarks.

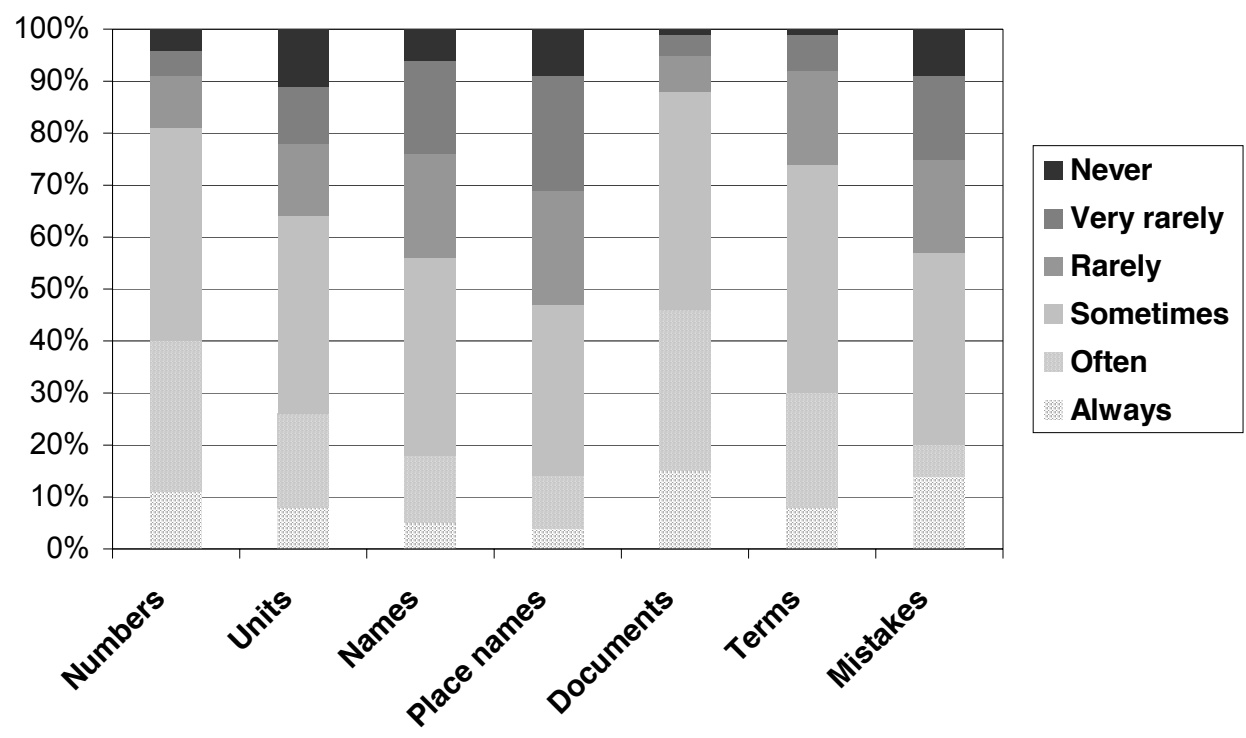

Figure 1. Expected assistance - aggregated results

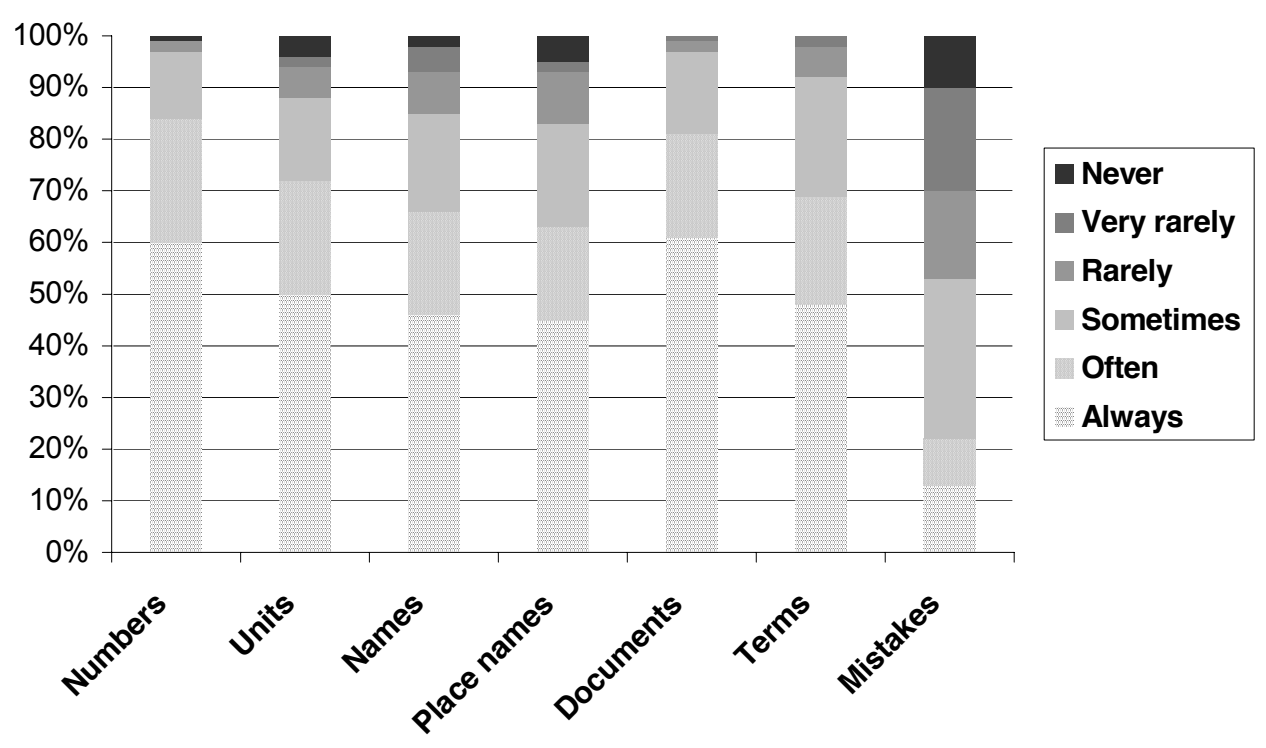

Figure 2. Assistance provided when asked - aggregated results

In general, some degree of cooperation is always present in interpretation 
booths, since absolutely negative replies ("never" answers) were in the minority, ranging from $0 \%$ (there are no respondents who provide no assistance in terminology and documentation) to $11 \%$ (that is the percentage of interpreters who never expect any assistance while processing units of measure).

\subsection{Off-mike Activities}

The nexts section of the questionnaire pertained to the off-mike activities of passive interpreters. It might be expected that an online survey will produce more candid responses than an interview since the respondent has a stronger feeling of anonymity. In fact, some respondents owned up to some quite interesting activities, described below.

Many interpreters say that they try to pay attention to what is going on when not interpreting, especially at the beginning of a conference, in order to acquire terminology (91\%), to learn as much about the conference as possible $(81 \%)$ and to assist their partners, if the need arises $(67 \%)$. The professionals surveyed are rarely too tired to listen when not interpreting (47\% of negative answers and $48 \%$ of "partially true" answers).

The respondents were also asked to select activities they engage in while offmike and then list additional activities not included in the potential answers. It appears that most interpreters use the time for last-minute preparation if documents are supplied shortly before the conference (92\%) and go through materials for the next speech they are about to interpret (90\%), which comes as no surprise.

To the author's surprise, as many as $45 \%$ of passive interpreters simply leave the booth for various reasons (to make phone calls, to smoke, to have some rest outside the conference hall). This means that in these cases there is no cooperation between the booth partners because the passive interpreter is simply not there. Fortunately, some respondents provided additional explanations. Some claim that whether or not they stay in the booth depends on the conference. If it is easy, the active colleague simply does not need any assistance. If it is difficult, it may be different: "I am $100 \%$ focused all the time and work almost as hard as the one who is interpreting", stated one respondent. Interestingly, another respondent declared that some conferences 
are too difficult to even offer any help, and all interpreters prefer to be left alone: "Any attempt at help from our partners would risk causing us to lose our thread." It seems that in some countries it is quite acceptable, or even required, to leave the booth while not interpreting, as shown by another respondent's comment: "Given that I work as a bilingual on the Paris market, I conform to the mores and habits which usually consist of leaving the colleague alone. Our work is usually so fast that it is almost impossible to help - by the time you write down something 3 sentences have gone by so it is really sink or swim! But most of us swim quite comfortably!"

Figure 3 presents answers to the question about off-mike activities.

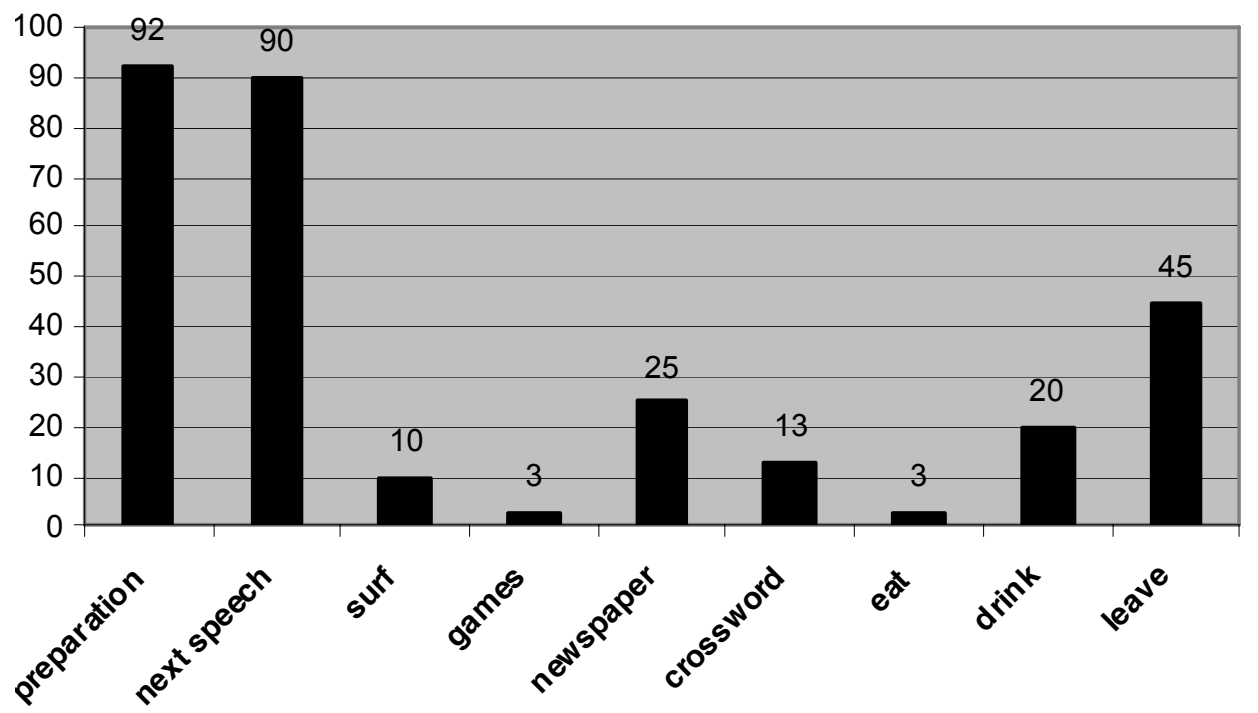

Figure 3. Activities of passive interpreters

Other activities listed by passive interpreters included the following:

- relax

- solve sudoku

- update glossaries

- knit, embroider mandalas 
- go for a smoke

- apply a mascara

- daydream

- return calls

- write letters by hand

- e-mail (in fact, one respondent sent an e-mail message saying that she would complete the questionnaire later since she was in the booth at that moment)

- sleep The results show that passive interpreters engage in various activities in the booth or outside it. However, it has to be borne in mind that some conferences are easy and experienced interpreters are qualified enough to accomplish the task without the boothmate's help and some conferences are extremely difficult and require such concentration that external assistance could actually be counter-productive. There are also markets where leaving the partner alone in the booth is a norm and a much expected behaviour, which is contrary to Jensen's findings regarding the American market. Across Languages and Cultures 9 (2) (2008)

\subsection{Turn-taking}

The respondents were also asked about the manner of turn-taking and the average duration of turns. The declared turn length varied from 15 to 45 minutes, with an average of 29 minutes. Thus, it can be concluded that the average uninterrupted period of work for a single interpreter is approximately half an hour. Interpreters prefer taking turns after a set period of time (57\% of "always" answers and 33\% of "sometimes" answers). It also sometimes happens that interpreters switch when speakers change (38\%) and when the active interpreter is tired $(40 \%)$. Figure 4 presents the detailed results of questions about turn-taking. 


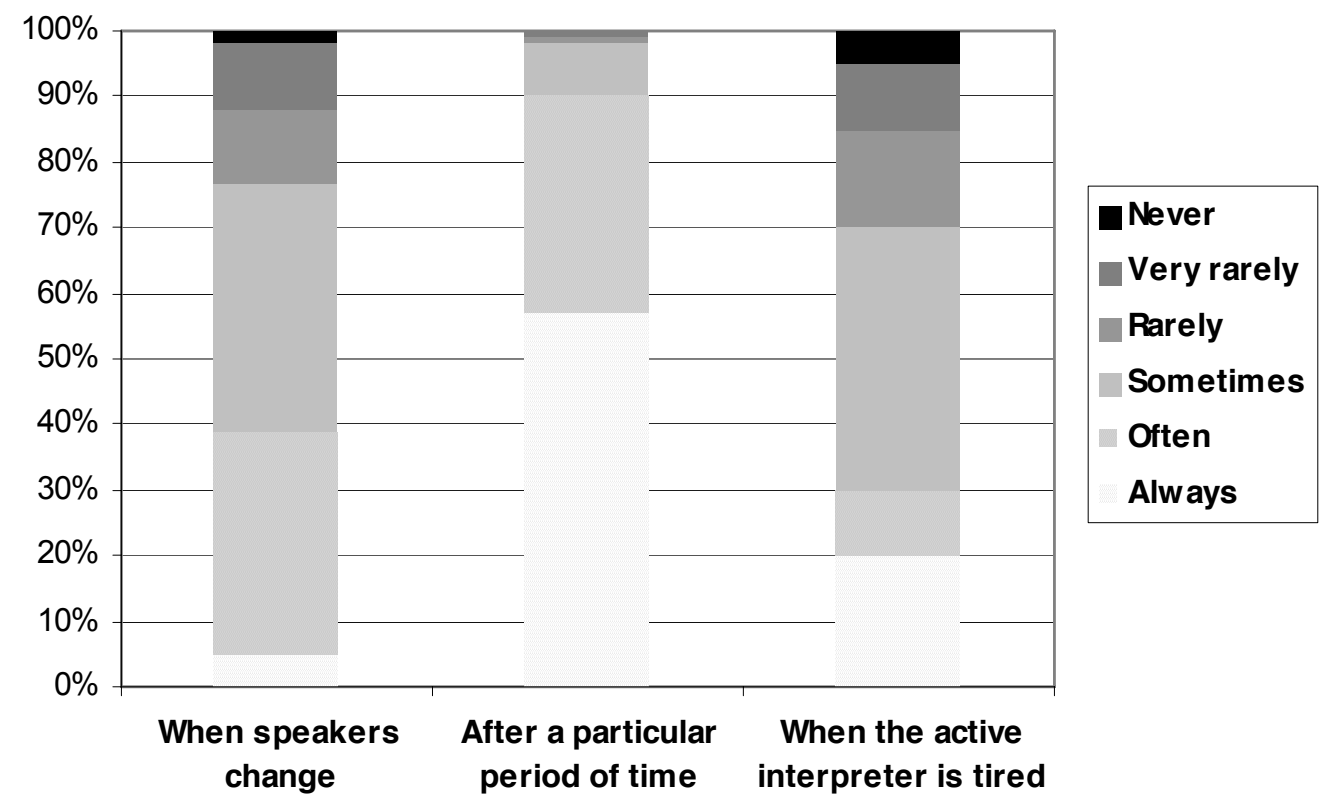

Figure 4. Turn-taking

\subsection{Teaching Cooperation}

Because the survey was conducted not only to examine the type and extent of actual cooperation in the booth, but also to collect data that could influence interpreter training programmes, the respondents were asked if turn-taking and cooperation in the booth should be taught in interpreting courses. The majority have received formal training in this field (82\%), and some respondents supplied additional comments showing that they work as trainers themselves. Thus, the responses to theses questions were very interesting from a didactic point of view. $81 \%$ of respondents claimed that turn-taking should be taught, and $92 \%$ claimed that cooperation should be taught in interpreting courses. Negative answers were $10 \%$ and $5 \%$, respectively. The remaining respondents chose the "I don't know" answer. Additional comments supplied voluntarily by some respondents show that many interpreters stress booth manners as an important element of team work and cooperation in the booth (for detailed comments from respondents see Appendix B). However, there are also interpreters who claim that the willingness to assist is a matter of personality rather than training. Moreover, one respondent who also works as an interpreter trainer claims that teaching 
This is an Author's Accepted Manuscript of an article in Across Languages and Cultures 9 (2), pp. 261-276 (2008), DOI: 10.1556/Acr.9.2008.2.6, available here:

http://www.akademiai.com/content/b512262w457x1210/?p=2ef008acd8774ea1b0a44cf0df68f970\&pi=5

cooperation in the booth is impractical since half of the trainees would have to be inactive rather than practice interpreting. On the other hand, the same respondent recalls offering some assisting exercises to interpreting trainees, for instance involving writing down names and numbers (see Appendix B for further comments).

Many interpreters participating in the survey used the text box at the end of the questionnaire form to supply additional comments. Most of them underlined the significant role of cooperation and teamwork in conference interpreting, in building a positive image of interpreters among conference delegates and recruiters and in successful completion of the task (see Appendix B for more details).

\section{CONCLUSIONS}

The study presented above aimed at examining the actual professional reality and working standards developed and accepted by communities of conference interpreters on various markets. By shedding more light on the doings of active and passive interpreters in the booth, specific conclusions can be drawn for the design of simultaneous interpreting classes and courses. The survey shows that most interpreters are willing to assist their booth colleagues when asked to and expect some assistance, especially as regards the processing of numbers or searching for documentation. Sometimes, however, any offer of help from the passive colleague may be counterproductive because it may disturb the concentration of the active colleague. Thus, the way assistance is offered is as important as the assistance itself.

The results of the study lead to the conclusion that we should teach cooperation in the booth, but it should be taught as an auxiliary skill only since other individually developed skills are much more important. It is useful to include in training courses some exercises in assisting. For instance, students should learn how to assist their colleagues interpreting difficult texts with a lot of non-contextual information such as statistics, names and dates. It would also be useful to introduce some exercises in turn-taking. The trainer can ask interpretation students to take turns very frequently in order to teach them how to reduce their EVS and which moment to choose to hand the microphone over to the colleague. Another useful suggestion is to organise 
This is an Author's Accepted Manuscript of an article in Across Languages and Cultures 9 (2), pp. 261-276 (2008), DOI: 10.1556/Acr.9.2008.2.6, available here:

http://www.akademiai.com/content/b512262w457x1210/?p=2ef008acd8774ea1b0a44cf0df68f970\&pi=5

simulated conferences so that students are exposed to working conditions similar to those in real life, in which booth partners have to rely on each other to be successful.

The survey showed that booth manners are of significance to practicing interpreters and should be stressed in training would-be interpreters. Students should be constantly reminded to use the microphone properly and not to generate too much noise in the booth (tapping, doodling, rustling papers). Booth manners and good habits in the booth should be developed in interpreting trainees from the very beginning. It is easy to acquire a good habit from the start than try to contain a bad habit later on, especially as improper booth behaviour and microphone management can have disastrous effects during a conference.

\section{REFERENCES}

AIIC Professional Standards. http://www.aiic.net/ViewPage.cfm/article122.htm (date of access: 15 March 2008).

Chesterman, A. 1997. Memes of Translation: The Spread of Ideas in Translation Theory. Amsterdam/Philadelphia: John Benjamins

Gile, D. 1995. Basic Concepts and Models for Interpreter and Translator Training. Amsterdam/Philadelphia: John Benjamins.

Gile, D. 1999. Testing the Effort Models' Tightrope Hypothesis in Simultaneous Interpreting - A Contribution. Hermes No. 23. 153-172.

Gillies, A. 2004. Conference Interpreting. A New Students' Companion. Cracow: Tertium. Jensen, J. B. 2006. The Strategic Partnership in the Conference Interpreting Booth. Paper

presented at the Annual Meeting of the American Translators Association.Jones, R. 2002. Conference Interpreting Explained. Manchester: St. Jerome Publishing.Kalina, S. 2001. Quality Requirements in Conference Interpreting. In: Proceedings of the 1st

International Conference on Translation and Interpretation Studies: Theories of Translation and Interpretation \& Problems in Korean Translation and Interpretation. Seoul: Graduate School of Interpretation and Translation, Hankuk University of Foreign Studies. 19-31.

Nolan, J. 2005. Interpretation. Techniques and Exercises. Clevedon/Buffalo/Toronto: Multilingual Matters.

Taylor-Bouladon, V. 2007. Conference Interpreting. Principles and Practice. Charleston: BookSurge Publishing. 


\section{Appendix A}

\section{QUESTIONNAIRE: COOPERATION IN THE BOOTH}

Dear Interpreter, please supply answers to the following questions about cooperation in a simultaneous interpretation booth (by putting an $\mathrm{X}$ next to the chosen answer or by entering relevant information). Your help is much appreciated.

Are you a freelance interpreter?

Yes No

How many years have you worked as a simultaneous interpreter? Have you received formal schooling in conference interpreting?

Yes No, I'm self-taught

Do you tend to work with the same colleague in the booth?

Yes No

Does it matter to you if you are to work with a colleague you don't know?

Yes No

DO YOU EXPECT YOUR BOOTH PARTNER TO ASSIST YOU IN INTERPRETATION BY:

- writing down numbers?

Always Often Sometimes Rarely Very rarely Never

- writing down units of measure?

Always Often Sometimes Rarely Very rarely Never

- writing down names?

Always Often Sometimes Rarely Very rarely Never

- writing down place names? 
Always Often Sometimes Rarely Very rarely Never

- searching for a document you need?

Always Often Sometimes Rarely Very rarely Never

- supplying a term that is missing in your output?

Always Often Sometimes Rarely Very rarely Never

- pointing out your mistakes?

Always Often Sometimes Rarely Very rarely Never

\section{DO YOUR OFFER ASSISTANCE TO YOUR BOOTH BY:}

PARTNERS, IF ASKED,

- writing down numbers?

Always Often Sometimes Rarely Very rarely Never

- writing down units of measure?

Always Often Sometimes Rarely Very rarely Never

- writing down names?

Always Often Sometimes Rarely Very rarely Never

- writing down place names?

Always Often Sometimes Rarely Very rarely Never

- searching for a document they need?

Always Often Sometimes Rarely Very rarely Never

- supplying a term that is missing in their output?

Always Often Sometimes Rarely Very rarely Never 
This is an Author's Accepted Manuscript of an article in Across Languages and Cultures 9 (2), pp. 261-276 (2008), DOI: 10.1556/Acr.9.2008.2.6, available here:

http://www.akademiai.com/content/b512262w457x1210/?p=2ef008acd8774ea1b0a44cf0df68f970\&pi=5

- pointing out their mistakes?

Always Often Sometimes Rarely Very rarely Never

WHAT ELSE DO YOU USUALLY DO WHEN YOUR BOOTHMATE IS INTERPRETING?

- last-minute preparation if documents are supplied shortly before the conference

- go through materials for the next speech you are about to interpret

- surf the Internet

- play computer games

- read a newspaper

- do a crossword puzzle

- eat

- drink

- leave the booth

- other things:

\section{TURN-TAKING}

Do you and your partner take turns in the booth when speakers change?

Always Often Sometimes Rarely Very rarely Never

- after a particular period of time?

Always Often Sometimes Rarely Very rarely Never

- when the active interpreter is tired? 
Always Often Sometimes Rarely Very rarely Never

How long is one turn if the same speaker speaks for more than an hour?

Do you think that turn-taking should be taught in interpreting courses?

Yes

No

I don't know

Do you think that cooperation in the booth should be taught in interpreting courses?

Yes

No

I don't know

\section{DIRECTIONALITY}

What is your B language?

Do you interpret into your B language?

What country are you based in?

How much do you interpret into your B language? (in \%)

\section{HOW TRUE ARE THESE STATEMENTS?}

I try to pay attention to speeches when I'm not interpreting in order to learn terminology (especially at the beginning of a conference).

Very true True Partially true Untrue

I try to pay attention to speeches when I'm not interpreting in order to know as much about the conference topic as possible.

Very true True Partially true Untrue 
I try to pay attention to speeches when I'm not interpreting in order to assist my booth partner, if needed.

Very true True Partially true Untrue

I am too tired to listen to speeches when I'm not interpreting.

Very true True Partially true Untrue

Other comments:

Thank you very much for your cooperation.

\section{Appendix B}

Additional comments to the questions about the need to teach turn-taking and cooperation in interpreting courses.

\section{WE SHOULD TEACH COOPERATION IN THE BOOTH}

- Good booth behaviour should be a topic at interpreting courses. Every conference is unique, and it is essential that booth mates cooperate. It enhances the quality of their interpreting.

- Booth manners should be taught in interpreting schools, especially how to limit noise levels for the working colleague and delegates (pouring water in glass close to microphone, turning pages too loudly, tearing pieces of paper, scribbling/doodling noisily, blowing nose/coughing when colleague is working, etc.).It is important for me that my partner is quiet when I am working (not coughing, opening plastic bags or rustling with a newspaper).

- People should certainly be taught booth manners and professional ethics, sometimes sorely missing in the free-lance world. Competitive colleagues tend to raise the level of stress in the booth.

WE SHOULD NOT TEACH COOPERATION IN THE BOOTH 
- Most of this is learnt on the job - not sure it needs teaching at a university.

- I do not think you can teach to cooperate. It is part of being a good person!

- Having taught interpreting myself, I do not consider this practical. Firstly, the academic hours are often too short. Secondly, it would require half the course to be inactive for too long. I did, however, include occasional hours of "assisting", i.e. writing down names, numbers, etc.

\section{COOPERATION IS CRUCIAL}

- This is teamwork. If the booth does badly, nobody would care to figure out, who of the colleagues is to blame, the whole booth or even team will get bad marks.

- In general, colleagues do not need much help. I do not like being helped when I do not need it, because you are diverted from your concentration for nothing. Sometimes I am happy if the colleagues can help out with things I didn't hear.

- Cooperation in the booth is a matter of intelligence and respect for the other. Intelligence, because it requires constant adaptation to the circumstances of the assignment. Respect for the other, because it requires the certainty that the boothmate is just as able as oneself to do a good job (or even better).

- The way the help is given is also VERY important: the other interpreter should NEVER whisper suggestions, as it is heard through the headphones by the audience, thus making them distrust the ability of the interpreters in the booth. The ideal help should be timely but in writing and always as subtle as possible so no one notices you or your partner was in trouble for a second or two.

- I tend to think that we work as a team in a booth and that the good performance and hence the public's/recruiter's/other booths' appreciation of our work is largely dependent on close collaboration and good relationships between colleagues in the same booth.

- Conference interpreting in the booth is a real teamwork. 